\title{
Catheter-directed thrombolysis in the treatment of acute deep venous thrombosis: a meta-analysis
}

\author{
J.J. Zheng', Z.H. Zhang', Z. Shan', W.J. Wang', X.X. Li' ${ }^{2}$, S.M. Wang', \\ Y.-X. $\mathrm{Li}^{1}$ and G.-S. Cheng ${ }^{3}$ \\ ${ }^{1}$ Cardiac Surgery, \\ The First Affiliated Hospital of Xi' an Jiaotong University School of Medicine, \\ Xi'an, Shaan Xi, China \\ ${ }^{2}$ Department of Vascular Surgery, \\ The First Affiliated Hospital of Zhongshan University, Guangzhou, \\ Guangdong, China \\ ${ }^{3}$ Second Department of Cardiology, \\ The First Affiliated Hospital of Xi'an Jiaotong University School of Medicine, \\ Xi'an, Shaan Xi, China \\ Corresponding author: J.J. Zheng \\ E-mail: JianJieZhengcn@163.com / shmingwang@126.com
}

Genet. Mol. Res. 13 (3): 5241-5249 (2014)

Received September 4, 2013

Accepted May 16, 2014

Published July 24, 2014

DOI http://dx.doi.org/10.4238/2014.July.24.1

ABSTRACT. We performed a meta-analysis for systematic evaluation of the status quo of catheter thrombolysis for the treatment of acute lower limb deep vein thrombosis in China. We searched the China Biomedical bibliographic database (CBM), China National Knowledge Infrastructure (CNKI), Weipu full-text electronic journals, Wanfang full-text database, and Medline (1990 through June 2011) for clinical randomized controlled trials of catheter-directed thrombolysis and superficial venous thrombolysis to compare their efficacies for the treatment of acute deep vein thrombosis. The results were analyzed by using the Cochrane-recommended RevMan 4.2 software package, and 
the odds ratio (OR) was used as the combined measure of efficacy. The search retrieved 8 randomized controlled trials, and meta-analysis using the total rate of effective treatment as the clinical observation index found that the combined OR for the catheter thrombolysis group versus the superficial venous thrombolysis group was significant $(\mathrm{P}<0.01$; $\mathrm{OR}=11.78 ; 95 \%$ confidence interval $=6.99-19.87)$. In conclusion, the meta-analysis indicated that catheter thrombolysis was more effective than superficial venous thrombolysis for the treatment of acute deep vein thrombosis in the lower limb in Chinese individuals. However, the included trials were only of medium quality, so more rational and scientific clinical trials are needed to validate this conclusion.

Key words: Deep vein thrombosis; Thrombolysis; Meta-analysis; Randomized controlled trial

\section{INTRODUCTION}

Deep vein thrombosis (DVT) is the abnormal coagulation of blood in deep veins, which can result in obstruction of the vena cava and venous disorders. If not promptly treated, DVT may be complicated by pulmonary embolism (PE) in the acute stage and in later stages may cause post-thrombotic syndrome (PTS), which can affect the patient's capacity for life and work. The incidence of DVT is high, and it is a common ailment. As the population's living standards increase and their diets change, and with advances in the technology used to detect blood vessel disease, the incidence of DVT increases annually, making it a major threat to both life and quality of life. Studies have shown that the annual incidence of symptomatic venous thromboembolism in the United States is about 71-117/100,000, of which 2/3 were DVT (White, 2003). Awareness of and efforts to prevent DVT in the United States have increased in recent years, but the incidence of DVT remains high, and approximately 275,000 patients are affected by DVT each year (Heit, 2008). Data from Sweden showed an incidence of DVT of 160/100,000 per year (Nordström et al., 1992). Less epidemiologic data are available concerning the incidence of DVT in China. Among 2742 patients presenting venous disease from September 1983 to April 2007 analyzed by the Shanghai Ninth People's Hospital, DVT accounted for $22.39 \%$ (Han et al., 2009). In recent years, a limited epidemiological survey showed a very high incidence of DVT with an increasing trend (Zhao et al., 2006).

The present treatments for DVT include surgery, anticoagulant therapy, and combined anticoagulant and thrombolytic therapy. In 1980, the U.S. National Institutes of Health suggested that thrombolytic therapy can be used as the standard treatment for acute DVT and PE. At that time it was used mainly for disease of the peripheral venous system. In 1994, Semba nd Dake reported that catheter-directed thrombolysis (CDT) produced good effects in patients with DVT. As interventional techniques have developed rapidly in recent years, CDT has become widely used, and the results of new clinical trials have been published frequently. Venography results after CDT indicate that it can achieve better short-term efficacy than anticoagulant therapy alone; it can also reduce the complications of DVT. The results from longterm follow-up indicate that CDT can significantly reduce the probability that PTS will occur after DVT (Manninen et al., 2012). 
Some researchers have reported that only $30 \%$ of patients with DVT can be treated with CDT (Gauci et al., 2011). Therefore, researchers from western countries have performed many controlled clinical studies on the efficacy of CDT in recent years, and meta-analyses of CDT-related articles have been reported. Although the conclusion remains controversial, meta-analysis has shown encouraging short-term effects of CDT (Patterson et al., 2010). Fewer such clinical trials have been performed in China. Therefore, we searched the China-related research literature and performed meta-analysis of the results in order to understand the therapeutic effect of CDT on acute lower limb deep vein thrombosis.

\section{MATERIAL AND METHODS}

\section{Search strategy}

The databases used included the China Biomedical bibliographic database (CBM), China National Knowledge Infrastructure (CNKI), Weipu full-text electronic journals, Wanfang full-text database, and Medline (1990 through June 2011), which were searched for clinical randomized controlled trials of catheter thrombolysis and superficial venous thrombolysis for the treatment of acute deep vein thrombosis in order to compare the efficacies of these procedures. The literature search was conducted on the subject words and key words, and the search terms included catheter thrombolysis and lower limb deep venous thrombosis.

\section{Inclusion criteria for studies}

The criteria for inclusion in the meta-analysis were consistency of the diagnostic criteria for disease diagnosis and of the definitions of the research factors; treatment of the patients in the test group with fibrinolytic therapy delivered via catheter into the site of acute lowerlimb deep venous thrombosis and of those in the control group with thrombolytic drugs via peripheral intravenous infusion; and a prospective, randomized, controlled trial (RCT) design.

\section{Collection and quality assessment of the data}

Two reviewers working independently screened and assessed the quality of the included studies, and the results were collected and cross-checked using a form designed to ensure that the data obtained from the documents were consistent. When disagreement occurred, a third researcher was brought in to discuss the issue until a solution was reached. The methodological quality of the included studies was evaluated by using the Jadad score, in which RCTs are scored on a scale of 1 to 5 .

\section{Statistical analysis}

The RevMan4.2 software provided by the Cochrane Collaboration network was used for statistical analysis of the data. RCTs with similar indices of efficacy were subjected to meta-analysis. First, the effect size of each trial was heterogeneity tested (Q-test) to calculate the odds ratio (OR) and 95\% confidence interval (CI) of the results. When the homogeneity of the test results is good, i.e., when $\mathrm{P}$ is $>0.1$ and $\mathrm{I}^{2}$ is $<50 \%$, a fixed-effect model can be selected. 
When the homogeneity is poor, a random-effect model can be selected and the merger effect estimated. Sensitivity testing and funnel plot assessment were used to check for publication bias.

\section{RESULTS}

\section{Inclusion and quality assessment of the relevant studies}

The searches initially retrieved 142 studies. First, the abstracts were read and studies other than randomized controlled trials, duplicate publications, and studies that did not meet the inclusion criteria were excluded. Finally, 8 randomized controlled trials that met the inclusion criteria were identified; these comprised 882 patients involved in randomized controlled clinical trials. The baseline conditions of the patients in the experimental and control groups were similar among the included trials, and the interventions were also parallel. The subjects of the 8 controlled trials were all Chinese, and the Jadad scores of the studies were 2 to 3 , which suggested that these were medium-quality studies. The randomization method in the eighth trial was not clear, and the follow-up method and number of and reasons for losses of patients to follow-up were not described in all trials. The characteristics of the studies are shown in Table 1.

\begin{tabular}{|c|c|c|c|c|c|c|}
\hline \multirow[t]{2}{*}{ Publication date } & \multirow[t]{2}{*}{ Author } & \multirow[t]{2}{*}{ Jaded scores } & \multicolumn{2}{|c|}{ CDT group } & \multicolumn{2}{|c|}{ Superficial vein thrombolysis group } \\
\hline & & & Effective cases & Total cases & Effective cases & Total cases \\
\hline $2005-5$ & Guan and Lin & 2 & 25 & 25 & 7 & 25 \\
\hline $2005-8$ & Gong et al. & 2 & 13 & 15 & 14 & 26 \\
\hline $2009-9$ & Yu et al. & 2 & 31 & 32 & 19 & 34 \\
\hline $2010-10$ & Zhang et al. & 3 & 187 & 195 & 62 & 102 \\
\hline 2011-1 & Li et al. & 2 & 23 & 24 & 36 & 56 \\
\hline 2011-3 & Zhou et al. & 3 & 23 & 24 & 34 & 36 \\
\hline 2011-4 & Luo and Zeng & 2 & 31 & 31 & 26 & 32 \\
\hline 2011-6 & Wang et al. & 3 & 67 & 71 & 107 & 154 \\
\hline
\end{tabular}

\section{Results of meta-analysis}

The total efficacy of the treatment was tested by using heterogeneity testing, which showed no statistically significant heterogeneity of the total efficacy $(\mathrm{Q}=3.99, \mathrm{P}>0.05, \mathrm{I} 2$ $=11.1 \%$ ) among the results of the included studies. As the total number from the combined results of multiple studies indicated homogeneity, a fixed-effect model could be used for the analysis. The total efficacy of CDT for the treatment of acute lower limb deep vein thrombosis was greater than that for superficial venous thrombolysis $(\mathrm{OR}=11.78 ; 95 \% \mathrm{CI}=6.99-19.87)$, indicating a statistically significant difference between the 2 treatments (Figure 1).

\section{Analysis of publication bias}

The funnel plot was drawn with the OR values of the total efficacies in the 8 trials on the abscissa and the reciprocals of the OR values on the vertical axis, and the non-symmetrical distribution of the resulting graph suggested the possibility of publication bias (Figure 2). 


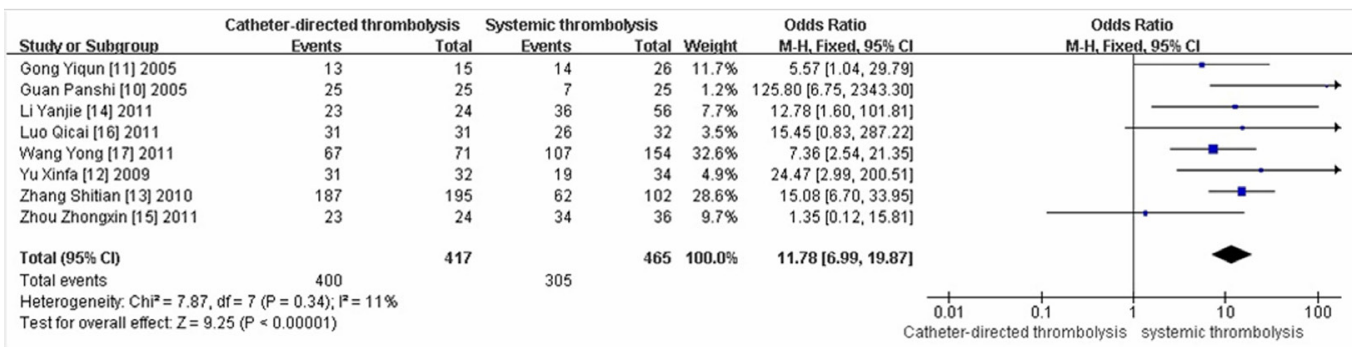

Figure 1. Comparison of total effective rates of catheter-directed thrombolysis (CDT) and superficial vein thrombolysis treating acute thrombosis of deep vein in lower limbs.

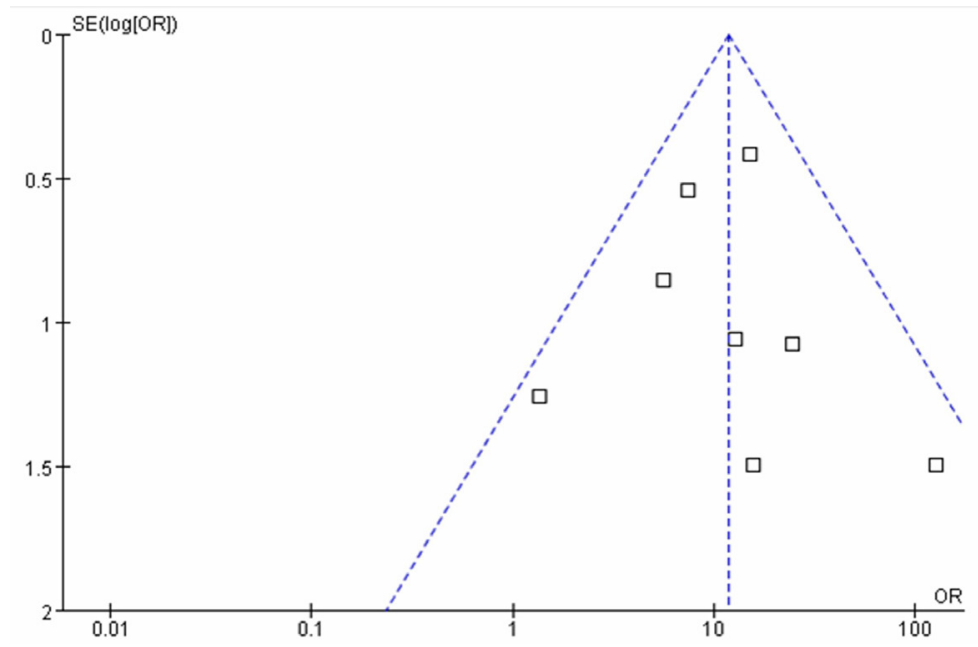

Figure 2. Analysis of publication bias on the selected literatures.

\section{Sensitivity analysis}

The data from Gong Yiqun's group were excluded because of the small sample size. The result of the sensitivity analysis of the data from the 7 remaining groups was $3.65(95 \% \mathrm{CI}$ $=2.45-5.42), \mathrm{Z}=5.56, \mathrm{P}<0.05$. The meta-analysis was repeated on the remaining data (402 cases in the CDT group and 439 in the superficial venous thrombolysis group), and the total OR $(95 \% \mathrm{CI})$ for effective treatment was 12.61 (7.25-21.92), $\mathrm{Z}=8.98, \mathrm{P}<0.0001$ (Figure 3).

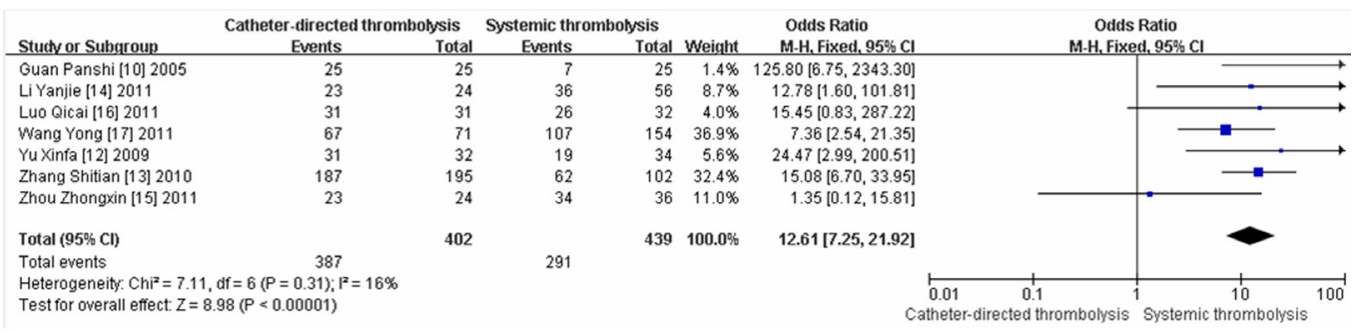

Figure 3. Sensitivity analysis of the selected literatures. 


\section{DISCUSSION}

DVT occurs frequently and can easily result in PTS: Kahn (2006) performed a comprehensive literature analysis and reported that 25 to $75 \%$ of cases of DVT result in PTS and 5 to $10 \%$ produce obvious clinical symptoms. Zhang and Jiang (2007) have analyzed the antegrade angiography results of 11,595 patients with chronic insufficiency of venous function in the deep veins of the lower limbs and find that 3269 cases (28.19\%) are due to PTS. PTS is the most serious complications of advanced DVT. In addition to obvious swelling of the affected limb and varicose superficial veins, chronic eczema, pigmentation, and even stasis ulcers can occur in the lower leg due to nutritional disorders of the skin. In severe cases, chronic nonhealing ulcers can affect the patient's quality of life and ability to work. Heit et al. (2001) reported that 170,000 people in the United States are affected by PTS each year and this disease is estimated to generate $\$ 200$ million in direct costs as well as additional indirect costs due to the loss of 2,000,000 working days each year.

Therefore, the goal of treatment of acute DVT in the lower extremity is to remove the thrombus as soon as possible, restore the blood flow in the deep vein, and preserve the maximum amount of valve function in order to prevent the occurrence of pulmonary embolism, reduce the incidence of PTS, prevent recurrence of thrombosis, and improve the patient's quality of life. The methods for treating DVT include anticoagulation, thrombolysis, surgical thrombectomy, and compression provided by elastic stockings. The question in each case is which treatment scheme will achieve the desired therapeutic goals. Although there is evidence that the incidence of PTS can be reduced by anticoagulant therapy for 3-6 months and elastic stocking therapy for 2 years, proximal DVT still leads to PTS in 50\% of patients (Kahn and Ginsberg, 2002) because anticoagulant therapy only inhibits further growth and prevents the recurrence of thrombus rather than rapidly dissolving the thrombus and thus protecting the venous valves against damage (Büller et al., 2004). Thrombolytic therapy is an effective way to relieve early-stage thrombosis, and regional or systemic thrombolysis via a superficial vein has been proven effective. However, because large doses of drugs are required for systemic thrombolysis, complications such as fatal visceral, cerebral, and/or retroperitoneal hemorrhage occur with some frequency, resulting in a high-risk benefit ratio for this therapy (Schweizer et al., 2000); therefore, the use of this modality is concerning.

CDT refers to fluoroscopy- or ultrasound-guided insertion of a thrombolysis catheter via the popliteal vein in the affected limb to the site of thrombosis to deliver a continuous infusion of thrombolytic drugs from a syringe pump directly to the thrombosis in order to dissolve it. In recent years, CDT has been increasingly adopted to treat acute deep vein thrombosis in the lower limb, and it is now used widely. A growing number of studies have shown the advantages of direct catheter thrombolysis. Enden et al. (2009) performed an RCT and showed that CDT combined with anticoagulant therapy significantly improved the rate of iliac and femoral vein patency over anticoagulant therapy alone. The number of published RCTs of CDT increased as European and American experts on the treatment of DVT rigorously evaluated the efficacy of CDT, and meta-analyses and systematic reviews of this topic have also been published (Janssen et al., 2005; Alesh et al., 2007; Vedantham, 2010). The results of these analyses have confirmed the efficacy of CDT, and Vedantham (2010) went so far as to state that CDT should be the preferred treatment for acute iliofemoral venous thrombosis. 
At present, the main modalities of thrombolytic therapy in China are peripheral superficial intravenous thrombolysis and CDT. Peripheral superficial venous thrombolysis refers to infusion of the thrombolytic drug through superficial veins in the instep or elsewhere; this was the earlier-developed method of thrombolysis. As the treatment of DVT in China has developed in recent years, the published literature on CDT has also increased. In this study, we performed meta-analysis of the literature published in China, and all of the subjects of the included studies were Chinese. All of the treatment in both CDT group and control group, including anticoagulation, antiplatelet adhesion and promoting blood circulation, which was achieved by Chinese medicine, were the same expected for thrombolysis route, although with the same thrombolysis medicine, urokinase. The results showed that the total rate of effective treatment for acute DVT of the lower limb was higher for CDT than for thrombolysis via superficial veins.

Thrombolytic therapy via peripheral superficial veins can partially remove fresh thrombus and thus reduce the incidence of PE, decrease limb swelling, and reduce the occurrence of PTS to some extent. However, its efficacy is limited by the limited contact between the thrombolytic drugs and the thrombus, and because most of the drug is injected directly into the systemic circulation through a collateral branch, the thrombolytic effect is often incomplete. In addition, the rate of dissolution of thrombi that completely block the blood vessel is less than $10 \%$, and as thrombosis is liable to recur, PTS still develops frequently. Furthermore, this treatment may take too long to complete and to take effect, the patient may not tolerate it, the dose of medication required is very large, and bleeding and other complications are liable to occur (Comerota and Aldridge, 1993). In contrast, the outstanding advantage of CDT is that the thrombolytic drugs are administered directly to the thrombus via the catheter, which increases the contact between the thrombolytic agents and thrombus, greatly increases the local concentration of thrombolytic drug to achieve the best thrombolytic effect in less time (thus reducing the time to thrombolysis), and maximizes the preservation of the deep venous valves; it can also promote compensation by collateral circulation and thus restore the patency of the venous trunk more quickly, and this improvement in venous return reduces venous pressure. The pain and edema symptoms are relieved, which is conducive to the recovery of the muscle pump function. The incidence of PTS is also greatly reduced. In addition, the dose of thrombolytic drugs that reaches the systemic circulation is significantly smaller, thereby reducing the rate of bleeding complications.

The majority of the trials did not describe the method of random allocation of the patients into the study groups, and few were double blind. Although most of the trials described the conditions of follow-up monitoring and the number of and reasons for losses of patients to follow-up, few statistics regarding or descriptions of bleeding resulting from thrombolysis or the occurrence of pulmonary embolism were mentioned in the included trials. The Jadad scores of the included trials were generally low, which affects to some extent the quality of the conclusions that can be drawn from this meta-analysis. The funnel plot of this study showed asymmetry, which suggests publication bias, and so more research is needed to provide evidence. We found fewer clinical trials of CDT than published in Europe and the United States, so no final conclusion that CDT is more effective than superficial venous thrombolysis for the treatment of DVT in Chinese people can be made on the basis of this meta-analysis, but the sensitivity analysis showed an OR $(95 \% \mathrm{CI})$ of 12.61 (7.25-21.92), $\mathrm{Z}=8.98, \mathrm{P}<0.0001$, which indicates that the analysis was still somewhat reliable. Several aspects of study design should be better emphasized in the future. First, the description of how the random allocation 
sequence was generated should be detailed; second, blind follow-up is required to collect valid data; and third, the numbers of patients lost to follow-up and the reasons for their loss must be described clearly to allow a more powerful conclusion. In addition, whether or not to place an inferior vena cava filter, the costs and benefits of CDT, and other controversial issues should be addressed in different prospective controlled studies to produce more comprehensive and credible conclusions.

\section{REFERENCES}

Alesh I, Kayali F and Stein PD (2007). Catheter-directed thrombolysis (intrathrombus injection) in treatment of deep venous thrombosis: a systematic review. Catheter. Cardiovasc. Interv. 70: 143-148.

Büller HR, Agnelli G, Hull RD, Hyers TM, et al. (2004). Antithrombotic therapy for venous thromboembolic disease: the Seventh ACCP Conference on Antithrombotic and Thrombolytic Therapy. Chest 126: 401S-428S.

Comerota AJ and Aldridge SC (1993). Thrombolytic therapy for deep venous thrombosis: a clinical review. Can. J. Surg. 36: 359-364.

Enden T, Kløw NE, Sandvik L, Slagsvold CE, et al. (2009). Catheter-directed thrombolysis vs. anticoagulant therapy alone in deep vein thrombosis: results of an open randomized, controlled trial reporting on short-term patency. J. Thromb. Haemost. 7: 1268-1275.

Gauci BN, Powell JT, Hunt BJ, Pilcher J, et al. (2011). The feasibility of catheter-directed thrombolysis for acute deep vein thrombosis: a regional perspective. Phlebology 26: 94-101.

Gong YQ, Gu YG, Chen ZH, Yu ZQ, et al. (2005). Endovascular thrombolysis in treatment of deep venous thrombosis. J. Chin. Phys. 8: 1101-1102.

Guan PS and Lin SM (2005). The therapeutic tactics for deep venous thrombosis in lower extremities (report of 50 cases). Chin. J. Thrombosis Hemostasis 5: 203-205.

Han WF, Huang XT, Yin MY, Lu M, et al. (2009). The clinical epidemiology of deep venous thromboembolism in the lower extremities. Chin. J. Gen. Surg. 24: 30-33.

Heit JA (2008). The epidemiology of venous thromboembolism in the community. Arterioscler. Thromb. Vasc. Biol. 28: 370-372.

Heit JA, Rooke TW, Silverstein MD, Mohr DN, et al. (2001). Trends in the incidence of venous stasis syndrome and venous ulcer: a 25-year population-based study. J. Vasc. Surg. 33: 1022-1027.

Janssen MC, Wollersheim H, Schultze-Kool LJ and Thien T (2005). Local and systemic thrombolytic therapy for acute deep venous thrombosis. Neth. J. Med. 63: 81-90.

Kahn SR (2006). The post-thrombotic syndrome: progress and pitfalls. Br. J. Haematol. 134: 357-365.

Kahn SR and Ginsberg JS (2002). The post-thrombotic syndrome: current knowledge, controversies, and directions for future research. Blood Rev. 16: 155-165.

Li YZ, Zhao ZF and Cui JG (2011). The efficacy analysis of catheter-directed thrombolysis for deep vein thrombosis. Chin. J. Curr. Adv. Gen. Surg. 1: 73-74.

Luo QC and Zeng QL (2011). Analysis the efficacy of implantation vena cava filter combined the catheterizing thrombolysis therapy in the treatment of deep venous thrombosis of lower-limb. Jiangxi Med. J. 4: 303-306.

Manninen H, Juutilainen A, Kaukanen E and Lehto S (2012). Catheter-directed thrombolysis of proximal lower extremity deep vein thrombosis: a prospective trial with venographic and clinical follow-up. Eur. J. Radiol. 81: 1197-1202.

Nordström M, Lindblad B, Bergqvist D and Kjellström T (1992). A prospective study of the incidence of deep-vein thrombosis within a defined urban population. J. Intern. Med. 232: 155-160.

Patterson BO, Hinchliffe R, Loftus IM, Thompson MM, et al. (2010). Indications for catheter-directed thrombolysis in the management of acute proximal deep venous thrombosis. Arterioscler. Thromb. Vasc. Biol. 30: 669-674.

Schweizer J, Kirch W, Koch R, Elix H, et al. (2000). Short- and long-term results after thrombolytic treatment of deep venous thrombosis. J. Am. Coll. Cardiol. 36: 1336-1343.

Semba CP and Dake MD (1994). Iliofemoral deep venous thrombosis: aggressive therapy with catheter-directed thrombolysis. Radiology 191: 487-494.

Vedantham S (2010). Catheter-directed thrombolysis for deep vein thrombosis. Curr. Opin. Hematol. 17: 464-468.

Wang Y, Zhang J, Wang SY, Duan ZQ, et al. (2011). Evaluation of efficacy of the anticoagulation and thrombolysis for deep venous thrombosis via local vein approach and peripheral vein approach. Zhonghua Wai Ke. Za Zhi. 49: 511-513.

White RH (2003). The epidemiology of venous thromboembolism. Circulation 107: I4-I8. 
Yu XF, Zhou CY and Chen XW (2009). Catheter-directed thrombolysis for lower extremity deep venous thrombosis. $J$. Southern Med. Univ. 9: 1930-1934.

Zhang PH and Jiang ME (2007). Clinical Vascular Surgery. 2nd edn. Science Press, Beijing.

Zhang ST, Sun CS and Yang X (2010). Percutaneous catheter thrombolytic therapy for acute iliofemoral venous thrombosis. Chin. J. Gerontol. 20: 2909-2911.

Zhao DM, Zhang ZD, Zhu R, Zheng Z, et al. (2006). The clinical epidemiology of inpatients with pulmonary thromboembolism for 32 years. Chin. J. Resp. Crit. Care Med. 5: 345-348.

Zhou ZX, Pan CQ, Fu FY, Lin ZQ, et al. (2011). Efficacy of regional administration of urokinase and argatroban via small saphenous vein catheter for treatment of acute deep venous thrombosis in the lower limb. Nan. Fang Yi Ke. Da. Xue Хие Вао 31: 539-543. 